\title{
Abrigo como medida de proteção para crianças e adolescentes: um levantamento bibliográfico PÓS-ECA
}

\section{Shelter like protection measure for children and adolescents: a bibliographical survey after the "estatuto da criança e do adolescente" (children and adolescent code)}

\author{
Ana Paula Serrata Malfitano ${ }^{1}$, Tatiana Viana da Silva ${ }^{2}$
}

http://dx.doi.org/10.11606/issn.2238-6149.v25i1p94-100

\begin{abstract}
Malfitano APS, Silva TV. Abrigo como medida de proteção para crianças e adolescentes: um levantamento bibliográfico PÓS-ECA. Rev Ter Ocup Univ São Paulo. 2014 jan./abr.;25(1):94-100.

RESUMO: O tema do abrigo e acolhimento institucional é de grande relevância social, naquilo que concerne as medidas de proteção à infância e à juventude. Tendo em vista conhecer o estado da arte acerca da produção acadêmica sobre temática de relevância social, objetivou-se examinar a produção científica sobre acolhimento e abrigamento de crianças e adolescentes, a partir da promulgação do Estatuto da Criança e do Adolescente (1990). Para tanto, realizou-se um levantamento em fontes bibliográficas de artigos, teses e dissertações, no período de 1990 a 2010. Foram encontrados 1.651 artigos, sendo que, após o refinamento, foram selecionados 44, que foram analisados segundo as categorias: base, descritor, ano, periódico, objetivo e população estudada. Em teses e dissertações, de um total de 6.228 encontradas, 500 estavam relacionados ao tema, sendo analisada sua quantidade por descritor. Conclui-se que, em 20 anos, o tema foi pouco pesquisado, demonstrando uma lacuna, do ponto de vista acadêmico-científico, para a apreensão e compreensão desse fenômeno social.
\end{abstract}

DESCRITORES: Criança; Adolescente; Defesa da criança e do adolescente; Bases de dados bibliográficas; Abrigo; Institucionalização; Adolescente institucionalizado; Criança institucionalizadainstitucional.
Malfitano APS, Silva TV. Shelter like protection measure for children and adolescents: a bibliographical survey after the "estatuto da criança e do adolescente" (children and adolescent code). Rev Ter Ocup Univ São Paulo. 2014 jan./abr.;25(1):94-100.

ABSTRACT: Shelter and institutional shelter are a subject with strong social importance because they are related with the protection to childhood and youth. With the goal to know the art state of the academic publication about the theme, this paper has the objective to examine the scientific production about reception and shelter to children and adolescents, after the 'Estatuto da Criança e do Adolescente' (Children and Adolescent Code) in Brazil. A research in articles and thesis databases was done, between 1990 and 2010. They were found 1.651 articles and, after the filter, they were selected 44 . They were analyzed about database, descriptor, year, journal, aim and population studied. In thesis, they were found 6.228 and 500 they were selected. The conclusion is that in 20 years the theme was poorly researched with gaps to understand the social question.

KEY WORDS: Child; Adolescent; Child advocacy; Databases, bibliographic; Shelter; Institutionalization; Adolescent, institutionalized; Child, institutionalized.

\footnotetext{
1. Professora Adjunta do Departamento de Terapia Ocupacional e do Programa de Pós-Graduação em Terapia Ocupacional da Universidade Federal de São Carlos (UFSCar).

2. Terapeuta ocupacional formada pela UFSCar.

Endereço para correspondência: Universidade Federal de São Carlos. Departamento de Terapia Ocupacional - Laboratório METUIA. Rodovia Washington Luís, km. 235, São Carlos-SP. CEP: 13565-905. E-mail: anamalfitano@ufscar.br
} 


\section{Abrigo e Institucionalização da Infância Pobre no Brasil: apontamentos históricos}

$\mathrm{O}$ abrigo, ou acolhimento institucional, é uma medida de proteção estabelecida pelo Estatuto da Criança e do Adolescente (ECA) que se destina a crianças e adolescentes afastados do convívio familiar, sendo definido com caráter provisório e excepcional, não implicando em privação de liberdade ${ }^{1}$. Deve ser a última medida de proteção a ser aplicada, sem mencionar as ações por adoção, pois rompe com os princípios anteriores do direito à família e à convivência com ela ${ }^{1}$.

Atualmente, em função de pesquisas anteriores que demonstraram o encaminhamento para abrigos como medida convencional, notoriamente ligada à pobreza de algumas famílias ${ }^{2}$, está em vigor o Plano Nacional de Promoção, Proteção e Defesa do Direito de Crianças e Adolescentes à Convivência Familiar e Comunitária ${ }^{3}$. O Plano propõe romper com a institucionalização daqueles sujeitos e promover, defender e garantir seu direito fundamental à convivência familiar e comunitária, estabelecendo diretrizes para que haja um reordenamento dos Serviços de Acolhimento Institucional integrado ao Sistema Único de Assistência Social (SUAS) ${ }^{4}$.

Tais leis visam à mudança de paradigma sobre a criança, a institucionalização e o abrigo como recurso de proteção e de cuidado. Assim, torna-se um elemento de relevância social a dedicação acadêmica para compreensão do acolhimento institucional e, por consequência, a produção do conhecimento sobre as intervenções que possam estar em desenvolvimento na sociedade brasileira, bem como os paradigmas em debate.

\section{OBJETIVOS}

Examinar a produção científica de artigos, dissertações e teses com a temática de abrigos e acolhimento de crianças e de adolescentes, a partir da reconfiguração legislativa inaugurada pelo ECA.

\section{MÉTODOS}

Realizou-se um levantamento em fontes bibliográficas de artigos, teses e dissertações, publicados no período de 1990 a 2010. Para as teses e dissertações, fez-se o recorte no período de 1990 a 2009, pois os dados de 2010 não estavam disponibilizados na ocasião da realização deste levantamento.

Utilizou-se as seguintes bases de dados eletrônicas: Portal de Teses e Dissertações da Coordenação de Pessoal de Nível Superior (CAPES), Portal de Periódicos da CAPES, Scientific Eletronic Library Online (SciELO), Biblioteca
Virtual de Saúde (BVS) e Biblioteca Virtual de Ciências Humanas (BVCH).

Os descritores utilizados para a efetivação da busca foram: "abrigo", "acolhimento à criança", "institucionalização", "orfanato", "convivência familiar e comunitária", "família substituta" e "família acolhedora". Foram escolhidos pelo tema (abrigo, acolhimento à criança) ou por alguma referência histórica (orfanato, institucionalização, convivência familiar e comunitária), ou ainda a uma outra alternativa de acolhimento para crianças e adolescentes, prevista pelas atuais leis brasileiras (família substituta e família acolhedora).

Os estudos foram pré-selecionados por meio dos títulos e das leituras dos resumos, com base no critério de inclusão: estudos referentes à temática de proteção à infầncia e adolescência. Foram excluídos textos que abordavam outras problemáticas, como o acolhimento institucional de outras populações, por exemplo, mulheres em situação de violência, assim como trabalhos em que o abrigo não era o tema principal de discussão.

Quando o título ou resumo não traziam informações suficientes, o texto foi lido na íntegra para avaliar sua inclusão ou não.

\section{RESULTADOS}

\section{Total dos artigos pesquisados nas bases de dados}

O levantamento totalizou 5.565 artigos. Foi aplicado o critério de inclusão, sendo, então, eliminados 5.457 textos. A maior parte dos textos eliminados referia-se a outras áreas do conhecimento, como as ciências biológicas e zoologia, os quais relatavam, por exemplo, o abrigo de componentes biológicos. Assim, o total de textos referentes a situações de proteção à criança e/ou ao adolescente, totalizou 107. Tais textos foram encontrados 29 na base SciELO, 30 no Portal de Periódicos da CAPES, 47 na Biblioteca Virtual de Saúde (BVS) e um na Biblioteca Virtual de Ciências Humanas (BVCH).

Dos 107 artigos relacionados ao tema, constatou-se que 63 estavam duplicados, por terem sido registrados em mais de uma base. Restando, portanto, o total de 44 textos.

Observando-se a predominância dos descritores, encontrou-se maior concentração em abrigo, com 55\%, e institucionalização, com $32 \%$. Os demais apresentavam pouca expressividade percentual, sendo, 5,6\% convivência familiar e comunitária, 2,8\% acolhimento à criança, 1,9\% família substituta, também 1,9\% para família acolhedora e, por fim, $0,8 \%$ orfanato. 
Tabela 1 - Número de artigos por descritor

\begin{tabular}{c|c|c|r|r|r|r|r}
\hline Base de Dados & $A$ & $B$ & $C$ & $D$ & $E$ & $F$ & $G$ \\
\hline SciELO & 16 & 8 & 0 & 0 & 2 & 2 & 1 \\
\hline Portal de Periódicos da CAPES & 16 & 13 & 0 & 0 & 0 & 0 & 0 \\
\hline BVS & 25 & 13 & 1 & 3 & 3 & 0 & 1 \\
\hline BVCH & 0 & 0 & 0 & 0 & 1 & 0 & 0 \\
\hline Total & 59 & 34 & 1 & 3 & 6 & 2 & 2 \\
\hline
\end{tabular}

$\mathrm{A}=$ Abrigo, $\mathrm{B}=$ Institucionalização, $\mathrm{C}=$ Orfanato, $\mathrm{D}=$ Acolhimento à criança, $\mathrm{E}=$ Convivência familiar e comunitária,

$\mathrm{F}=$ Família substituta, $\mathrm{G}=$ Família acolhedora

Fazendo-se um levantamento sobre os anos de publicação dos artigos, observa-se a inexistência de textos sobre a temática entre 1990 e 1998, sendo o primeiro de 1999. Encontra-se uma distribuição não equânime, sendo que os anos de 2004, 2007, 2008 e 2009 possuem uma maior quantidade de textos publicados.

\section{Um olhar mais detalhado sobre a temática dos artigos produzidos}

Os artigos encontrados (Tabela 2) demonstram que os estudos sobre abrigo para crianças e adolescentes não estão direcionados para um objetivo em comum. O foco dos estudos é bastante variado, sendo que foi realizada a seguinte categorização, seguida do número de artigos publicados: aspectos psicológicos (11), família (10), funcionamento do abrigo (8), experiência dos abrigados (4), desenvolvimento infantil (3), saúde (2), cuidadores/monitores das instituições (1), adoção (1), políticas públicas de assistência em orfanato/abrigo (1), outros (3).

Tabela 2 - Classificação dos artigos publicados

\begin{tabular}{|c|c|c|c|c|}
\hline Título & Periódico & Ano & $\begin{array}{l}\text { População- } \\
\text { alvo }\end{array}$ & $\begin{array}{l}\text { Classifica- } \\
\text { cão** }\end{array}$ \\
\hline $\mathrm{O}$ auto-retrato em crianças institucionalizadas & Paidéia & 1999 & 1 & A \\
\hline $\begin{array}{l}\text { Vivendo em família ou em instituição: a experiência ótima, metas e desafios na } \\
\text { vida de adolescentes }\end{array}$ & Paidéia & 2000 & 2 & B \\
\hline Conhecendo a experiência de crianças institucionalizadas & Rev. esc. enferm. USP & 2001 & 3 & $\mathrm{~B}$ \\
\hline Crianças em situação de rua falam sobre os abrigos & Rev. esc. enferm. USP & 2002 & 3 & $\mathrm{~B}$ \\
\hline $\begin{array}{l}\text { Habilidades de pensamento criativo em crianças institucionalizadas e não } \\
\text { institucionalizadas }\end{array}$ & Estud. psicol. & 2003 & 3 & A \\
\hline $\begin{array}{l}\text { Intervenção em terapia ocupacional em casas-lares com crianças pré-escolares } \\
\text { vítimas de violência doméstica: relato de experiência }\end{array}$ & Cader. Ter. Ocup. UFSCar & 2003 & 3 & $\mathrm{C}$ \\
\hline $\begin{array}{l}\text { O abrigo para crianças e adolescentes: considerações acerca do papel do } \\
\text { terapeuta ocupacional }\end{array}$ & Rev. Ter. Ocup. USP & 2003 & 4 & $\mathrm{O}$ \\
\hline A subjetividade presente no estudo psicossocial da adoção & Psicol. Reflex. Crit. & 2004 & 5 & $\mathrm{D}$ \\
\hline $\begin{array}{l}\text { Análise documental e contexto de desenvolvimento: estatutos de uma } \\
\text { instituição de proteção à infância de Salvador, Bahia }\end{array}$ & Estud. psicol. (Campinas) & 2004 & 4 & $\mathrm{C}$ \\
\hline $\begin{array}{l}\text { Avaliação e diagnóstico do processo de reinserção familiar e social de crianças } \\
\text { e adolescentes egressos de uma casa de passagem }\end{array}$ & Cogitare enferm & 2004 & 6 & $\mathrm{E}$ \\
\hline Brincando de casinha: significado de família para crianças institucionalizadas & Estud. psicol. (Natal) & 2004 & 3 & E \\
\hline Depressão e desempenho escolar em crianças e adolescentes institucionalizados & Psicol. Reflex. Crit. & 2004 & 3 e 2 & A \\
\hline $\begin{array}{l}\text { Estudo sociométrico de uma instituição alternativa para crianças e adolescentes } \\
\text { em situação de rua: construindo uma proposta pedagógica }\end{array}$ & Psicol. reflex. Crit & 2004 & 3 e 2 & $\mathrm{C}$ \\
\hline
\end{tabular}


Malfitano APS, Silva TV. Abrigo como medida de proteção para crianças. Rev Ter Ocup Univ São Paulo. 2014 jan./abr.;25(1):94-100..

Continuação

Os efeitos do abandono para o desenvolvimento psicológico de bebês e a maternagem como fator de proteção

Relação de apego entre crianças institucionalizadas que vivem em situação de abrigo

O significado do abandono para crianças institucionalizadas

A rede de apoio social e afetivo de adolescentes institucionalizados no Sul do

Brasil

O impacto da institucionalização na infância e na adolescência: uma revisão de

literatura

Abrigo para crianças de 0 a 6 anos: um olhar sobre as diferentes concepções e

suas interfaces

Abrigos para crianças vítimas de violência doméstica: funcionamento relatado

pelas crianças e pelos dirigentes

Efeitos de um programa de práticas educativas para monitoras de um abrigo infantil

Institucionalização precoce e prolongada de crianças: discutindo aspectos decisivos para o desenvolvimento

Interações afetivas de crianças abrigadas: um estudo etnográfico

Políticas públicas para adolescentes em vulnerabilidade social: abrigo e provisoriedade

Retornando para a família de origem: fatores de risco e proteção no processo de reinserção de uma adolescente institucionalizada

Sintomas depressivos e eventos estressores em crianças e adolescentes no

contexto de institucionalização

Assistência em saúde às crianças e adolescentes abrigados em Ribeirão Preto, SP

Abrigar/desabrigar: conhecendo o papel das famílias nesse processo

Adolescência e institucionalização numa perspectiva de vinculação

Apego em adolescentes institucionalizadas: processos de resiliência na formação de novos vínculos afetivos

Família substituta: uma proposta de intervenção clínica na adoção tardia

Mãe é a que cria: o significado de uma maternidade substituta

O momento da saída do abrigo por causa da maioridade: a voz dos adolescentes

O olhar da psicologia no abrigo: uma cartografia

Um olhar sobre o abrigamento: a importância dos vínculos em contexto de abrigo

Acolhimento familiar: uma alternativa de proteção para crianças e adolescentes

Construção das dificuldades de aprendizagem em crianças adotadas

O abrigamento e as redes de proteção para a infância e a juventude

O abrigo como fator de risco ou proteção: avaliação institucional e indicadores

de qualidade

Processos de saúde e doença entre crianças institucionalizadas: uma visão ecológica

A prática educativa de profissionais cuidadores em abrigos: enfrentando a

violência vivida por mulheres adolescentes

Um estudo ecológico sobre as interações da família com o abrigo

Vulnerabilidades de adolescentes com vivências de rua

A convivência familiar e comunitária é direito da criança e do adolescente: uma realidade a ser repensada pela escola

Casa abrigo: a solução ou o problema?

Processo de reinserção familiar: estudo de casos de adolescentes que viveram em instituição de abrigo

\begin{tabular}{|c|c|c|c|}
\hline Estud. psicol. (Campinas) & 2004 & 3 & A \\
\hline Psicol. estud. & 2004 & 3 & A \\
\hline $\begin{array}{l}\text { Revista Interamericana de } \\
\text { Psicología }\end{array}$ & 2005 & 3 & A \\
\hline $\begin{array}{l}\text { Revista Interamericana de } \\
\text { Psicología }\end{array}$ & 2006 & 2 & B \\
\hline Psicol. Soc. & 2006 & 4 & $\mathrm{~N}$ \\
\hline Rev. mal-estar subj & 2007 & 3 & $\mathrm{C}$ \\
\hline Psicol. Teor. prát & 2007 & 3 & $\mathrm{CF}$ \\
\hline $\begin{array}{l}\text { Rev. bras. ter. comport. } \\
\operatorname{cogn}\end{array}$ & 2007 & 5 & $\mathrm{~F}$ \\
\hline Aletheia & 2007 & 4 & G \\
\hline Ciênc. saúde coletiva & 2007 & 3 & $\mathrm{~B}$ \\
\hline Psicol. ciênc. prof & $\underline{2007}$ & 4 & $\mathrm{H}$ \\
\hline $\begin{array}{l}\text { Rev. bras. crescimento } \\
\text { desenvolv. Hum }\end{array}$ & 2007 & 4,5 e 6 & E \\
\hline Rev. psiquiatr. Rio Gd. Sul & 2007 & 2 & A \\
\hline Rev Bras Enferm & 2008 & 4 & I \\
\hline Estud. psicol. (Campinas) & 2008 & 6 & $\mathrm{E}$ \\
\hline Psicol. Soc & 2008 & 2 & A \\
\hline Psico & 2008 & 2 & A \\
\hline Psicol. teor. Prat & 2008 & 3 & $\mathrm{E}$ \\
\hline Estud. psicol. & 2008 & 2 e 6 & $\mathrm{E}$ \\
\hline Psicol. rev. & 2008 & 2 & $\mathrm{O}$ \\
\hline Psicologia \& Sociedade & 2008 & 4 & $\mathrm{C}$ \\
\hline Psicol. esc. educ. & 2008 & 5 & A \\
\hline Psicol. reflex. Crit & 2009 & 4 & $\mathrm{E}$ \\
\hline Cad. Pesqui. & 2009 & 3 & $\mathrm{G}$ \\
\hline Fractal, Rev. Psicol. & 2009 & 3 e 2 & G \\
\hline Psicol. Reflex. Crit. & 2009 & 5 & $\mathrm{C}$ \\
\hline Ciênc. saúde coletiva & 2009 & 3 & I \\
\hline Rev. Latino-Am. Enferm & 2009 & 5 & A \\
\hline Paidéia & 2009 & 5 e 6 & $\mathrm{E}$ \\
\hline $\begin{array}{l}\text { Esc. Anna Nery Rev. } \\
\text { Enferm }\end{array}$ & 2009 & 2 & $\mathrm{O}$ \\
\hline Cad. CEDES & 2010 & 4 & $\mathrm{E}$ \\
\hline Psicol. teor. pesqui & 2010 & 3 & $\mathrm{C}$ \\
\hline Estud. psicol. (Natal) & 2010 & 2 & E \\
\hline
\end{tabular}

* 1 = Crianças institucionalizadas e não institucionalizadas, $2=$ Adolescentes, $3=$ Crianças, $4=$ Não especificado, 5 = Técnicos, $6=$ Famílias. **A= Aspectos Psicológicos, $\mathrm{B}=$ Experiência dos abrigados, $\mathrm{C}=$ Funcionamento do abrigo, $\mathrm{D}=$ Adoção, $\mathrm{E}=$ Família, $\mathrm{F}=$ Cuidadores/monitores, $\mathrm{G}=$ Desenvolvimento Infantil, $\mathrm{H}=$ Política, $\mathrm{I}=$ Saúde, $\mathrm{O}=$ Outros, $\mathrm{N}=$ Não se aplica. 
Dos artigos encontrados com o objetivo de estudar aspectos psicológicos das crianças e adolescentes abrigados, os enfoques percorreram os seguintes temas, segundo denominação dos próprios autores dos artigos em estudo: imagem corporal e autoconceito, reorganização da esfera emocional, representação do apego, manifestação de sintomas depressivos e impacto de eventos estressores, modos de subjetivação, sentidos produzidos pela saída do abrigo, ajustamento psicológico e significado de abandono.

Quatro artigos abordavam o impacto das situações vivenciadas pelas crianças e adolescentes em seu processo de desenvolvimento, classificados aqui como "experiência dos abrigados". Os temas foram: desenvolvimento geral, distúrbio depressivo e desempenho escolar, dificuldades de aprendizagem e pensamento criativo. Sendo que dois artigos faziam a comparação entre o desenvolvimento dos institucionalizados e dos não institucionalizados.

A saúde foi objeto de dois manuscritos, sendo um sobre processos de saúde e doença das crianças e adolescentes abrigados e outro sobre a assistência em saúde em abrigos.

A perspectiva sobre o funcionamento do abrigo foi classificada em oito artigos, com os temas: rotina de funcionamento, principais elementos responsáveis pela fixação e adesão, práticas cotidianas e estrutura física, referenciais para orientação de funcionamento e estratégias para avaliação do trabalho desenvolvido nos abrigos. Ainda, havia um artigo sobre os monitores/cuidadores que trabalham na instituição, abordando a elaboração e avaliação de treinamento, as interações entre monitores e as crianças e as estratégias utilizadas na atenção às situações de violência.

Tendo em vista o longo período de funcionamento dessas instituições e sua recente mudança na filosofia e estruturação, estudar o atual funcionamento de abrigos e as relações estabelecidas nele torna-se fundamental para que sejam compreendidas como (e se) estão sendo efetivadas tais mudanças. Destaca-se que dois artigos enfocaram as mudanças históricas das medidas de assistência a essa população no Brasil, a fim de dar visibilidade às práticas produzidas, e apenas um artigo referia-se diretamente aos desafios que o Plano Nacional de Convivência Familiar e Comunitária encontrará para ser efetivado.

Em relação ao retorno ou a substituição temporária da família, encontraram-se três sobre reinserção familiar, três sobre adoção, dois sobre acolhimento familiar e quatro sobre família das crianças e adolescentes abrigadas, em geral. Os textos sobre reinserção familiar referiam-se ao retorno à família de origem por adolescentes. Podem ser levantadas

(1) Disponível em: http://www.capes.gov.br/servicos/banco-de-teses algumas hipóteses sobre esse recorte, devido às dificuldades sabidas para a adoção de adolescentes, prevalecendo a preferência por crianças mais novas, juntamente com poucas experiências dedicadas à colocação dessa faixa etária em famílias substitutas.

Nos textos sobre adoção, um se referia a bebês. Nos outros dois, um se referia a relato de caso de uma criança de cinco anos e o outro sobre os critérios elaborados para estudos psicossociais para o processo de adoção.

Predomina, nos artigos examinados, diversas problemáticas em torno das crianças e adolescentes abrigados, porém poucos trabalhos dedicados à compreensão e produção de conhecimento sobre os aspectos relacionados à prática propriamente dita do acolhimento institucional, medida de proteção de extrema relevância para a população infantojuvenil brasileira.

\section{Teses e Dissertações disponíveis no Portal CAPES}

Para o levantamento de teses e dissertações defendidas em Programas de Pós-Graduação no país, utilizamos o Banco de Teses da $\mathrm{CAPES}^{(1)}$, local em que se encontra o registro dos resumos de todas as teses e dissertações de Programas de Pós-Graduação reconhecidos e avaliados pelo órgão.

Encontramos o total de 6.228 resumos, no período de 1990 a 2009. Igualmente ao levantamento de artigos, aplicou-se o critério de inclusão, restando 500 dissertações ou teses relacionadas ao acolhimento institucional de crianças e/ou adolescentes. Quanto aos descritores, foram encontradas 221 teses ou dissertações com o descritor abrigo; 135 com institucionalização; 4 com orfanato; 60 com acolhimento à criança; 31 com convivência familiar e comunitária; 40 com família substituta e nove com família acolhedora.

O descritor abrigo corresponde a 44,2\% dos resumos selecionados e institucionalização, a 27\%. Na sequência, acolhimento à criança, com $12 \%$, família substituta, com $8 \%$, convivência familiar e comunitária, com $6,2 \%$, família acolhedora, com $1,8 \%$ e orfanato com $0,8 \%$.

Quanto à distribuição por ano, encontrou-se trabalhos realizados desde 1990. A maior concentração estava nos anos de 2008, 2006 e 2009 respectivamente.

\section{Discutindo alguns aspectos dos achados}

\section{A Produção no Tempo}

Os textos encontrados apareceram em uma crescente 
inconstante durante os 20 anos pesquisados.

Quanto às teses e dissertações, observa-se que a temática integra o campo da Pós-Graduação desde 1990, sendo que na primeira metade da década houve uma média de 1,6 trabalhos por ano, crescendo consideravelmente nos anos seguintes, entre 1996 e 1999, para 13,25 trabalhos anuais. Tal quadro é condizente com os fatos históricos, uma vez que o ECA foi promulgado em 1990 e as discussões sobre o abrigo, com essa nomenclatura, são realizadas a partir de então, tendo como reflexo o aumento das produções na segunda metade da década de 1990. Nos anos 2000, as teses e dissertações apresentaram um aumento progressivo, tendo como exceção os anos de 2002, 2007 e 2009, os quais tiveram queda.

Já a produção em artigos não apresenta a mesma crescente, demonstrando a pequena inserção do tema no campo dos periódicos científicos. O primeiro artigo encontrado é de 1999 e permanece com a média de um texto por ano até 2003. A partir de 2004 há um aumento, porém não mantém uma progressão constante nos anos.

Apesar de a efetivação dos direitos da criança e do adolescente ser prioridade absoluta determinada por lei, juntamente à mudança relativamente recente na estruturação de tais serviços, assim como pela complexidade da ação de acolhimento, como medida protetiva para as crianças e os adolescentes que tiveram seus direitos violados ou ameaçados, a porcentagem de artigos, teses e dissertações encontradas demonstra que o assunto não tem sido prioridade nos estudos científicos, resultando em pouco material que poderia oferecer subsídios para a efetivação e aprimoramento dos serviços.

\section{Descritores}

O descritor abrigo apresentou a maior quantidade de trabalhos encontrados, tanto em relação aos artigos quanto às teses e dissertações, conforme esperado.

O termo institucionalização foi o segundo mais encontrado nos trabalhos. A institucionalização foi amplamente discutida na década de 1980, principalmente no campo da saúde mental. Acredita-se que, uma década depois, haja reflexo dessa discussão na produção acadêmica. Não se observa, na literatura do campo da assistência social, uma tradição dessa discussão, sendo de grande relevância que esse descritor apareça também associado a temas em torno dos abrigos.

Com o descritor acolhimento à criança foram encontrados três artigos, sendo que todos se referiam ao processo de acolhimento em saúde. Já nas teses e dissertações, o descritor esteve presente em 60 trabalhos, variando as abordagens em torno da discussão do acolhimento, incluindo relações mais estritas ao processo em abrigos.

Com os descritores família substituta e família acolhedora foram encontrados dois artigos. Entre as teses e dissertações foram encontradas 49 relacionadas à temática. Nota-se que ainda há pouca pesquisa e ações documentadas em relação ao assunto, recentemente legislado no Brasil ${ }^{3,4}$. O questionamento levantado é se essas alternativas de proteção estão sendo efetivadas nacionalmente e têm sido pouco pesquisadas ou se não estão sendo implementadas nos municípios. Tal discussão é de extrema relevância no momento atual, em que os esforços políticos e legislativos se encontram para a mudança do paradigma da proteção, deslocando-se da proposta de acolhimentos em instituições ou adoção para o acolhimento provisório em famílias substitutas. Pela relevância social e poucas investigações realizadas, trata-se de uma área que requer aprofundamento para pesquisas e intervenções.

Por fim, o descritor orfanato foi encontrado apenas em um artigo e em quatro teses e dissertações, o que sinaliza a efetivação da mudança de termo para abrigo ou acolhimento, conforme promulgado pelo ECA. A modificação do termo torna-se necessária como parte das estratégias de concepção ideológica e prática sobre ações cabíveis a esse tipo de instituição.

\section{As Bases de Dados}

Encontrou-se uma maior quantidade de manuscritos na Biblioteca Virtual de Saúde, resultando em 47 artigos, ou seja, 44\%. Tal dado demonstra a preponderância da área de saúde para a indexação de textos sobre a temática.

Esperava-se que a discussão sobre abrigos para crianças e adolescentes estivesse, com maior ascendência, no campo das ciências humanas, causando estranhamento a filiação prioritária de trabalhos na saúde. Entretanto, a pesquisa na Biblioteca Virtual de Ciências Humanas resultou em apenas um artigo, registrado com o descritor convivência familiar e comunitária. Tal fato requer um maior aprofundamento de estudo, desde a questão de a base de dado escolhida para a pesquisa, na compreensão sobre sua abrangência e representatividade nas Ciências Humanas, partindo, se pertinente, para a investigação sobre os motivos que fazem com que o tema abrigo tenha sido e esteja pouco pesquisado na área, nos últimos vinte anos.

Analisando-se a área de conhecimento dos periódicos com artigos publicados, encontrou-se um predomínio 
das revistas de Psicologia, em diferentes subáreas do conhecimento. Aárea de Psicologia totalizou 30 artigos, dos 44 estudados, ou seja, $69 \%$. Destes, 23 estão em revistas de psicologia que não especificam uma subárea, quatro em psicologia e educação e três em psicologia social. Posteriormente, aparecem as áreas de Enfermagem, com $12 \%$, Educação, Psiquiatria, Saúde Coletiva e Terapia Ocupacional, com $4 \%$ cada.

A predominância da área de Psicologia pode estar associada a sua participação como categoria na equipe de referência dos serviços de acolhimento, conforme definido pela Norma Operacional Básica de Recursos Humanos do Sistema Único da Assistência Social (NOB/RH-SUAS) ${ }^{5}$. Possivelmente, a maioria dos abrigos atuais funciona com a participação desses profissionais e, portanto, é importante que deem continuidade às pesquisas e estudos sobre o tema.

Porém, à luz desse argumento, surge o questionamento sobre a produção no âmbito da área de Assistência Social, na medida em que também é um profissional previsto para atuação em tais espaços e, mais ainda, com histórico profissional arraigado nessas instituições. Seria relevante, portanto, conhecer as causas de não se ter, nos últimos vinte anos, nenhuma revista do serviço social com divulgação de

\section{REFERÊNCIAS}

1. Brasil. Lei $\mathrm{n}^{\circ} .8069$, de 13 de julho de 1990. Dispões sobre o Estatuto da Criança e do Adolescente e dá outras providências. Obtido em 15 de dezembro, 2011. Disponível em: http://www. planalto.gov.br/ccivil_03/leis/L8069.htm.

2. Silva ERA, organizador. (2004). O direito à convivência familiar e comunitária: os abrigos para crianças e adolescentes no Brasil. Brasília: IPEA/CONANDA; 2004. Disponível em: http://www. ipea.gov.br/005/00502001.jsp?ttCD_CHAVE=245.

3. Brasil. Ministério do Desenvolvimento Social e Combate à Fome, Secretaria Especial dos Direitos Humanos. Plano conhecimento com os descritores utilizados para a presente pesquisa.

\section{CONSIDERAÇÕES FINAIS}

A mudança de paradigma em torno da criança e do adolescente no Brasil, na dimensão dos direitos e da cidadania, além de seu lugar de prioridade absoluta, traz a relevância social da temática, inclusive para o universo acadêmico e para a produção de conhecimento sobre esse público, suas vivências e a efetividade social dos princípios legislativos estabelecidos.

No âmbito da configuração da sociedade brasileira, assinala-se que, após mais de vinte anos de promulgação do ECA, muitas discussões são ainda necessárias para que se compreenda a efetivação do princípio jurídico nas vivências sociais.

Destaca-se a necessidade que a academia se debruce sobre a realidade de acolhimento às crianças e adolescentes em instituições, com necessidade de compreensão, aprofundamento e produção de conhecimento que se traduzam em tecnologias sociais aplicáveis aos cenários sociais concretos. nacional de promoção, proteção e defesa do direito de crianças e adolescentes à convivência familiar e comunitária. Brasília; 2006

4. Brasil. Ministério do Desenvolvimento Social e Combate à Fome, Secretaria Nacional de Assistência Social. Orientações técnicas: serviços de acolhimento para crianças e adolescentes. Brasília; 2009.

5. Brasil. Ministério do Desenvolvimento Social, Secretaria Nacional de Assistência Social. Norma operacional básica de recursos humanos do SUAS (NOB/RH/SUAS). Brasília; 2006. 